\title{
Theorem of (upwards) Harmonic Subdivision
}

\author{
Demetrios E. Lekkas \\ Ex Academic, The Hellenic \\ Open University, Patras Greece \\ email:ja-dim@hotmail.com
}

$\mathrm{T}$

he purpose of this paper is to study the theoretical outcome of an aliquot equidistant fingering / fretting pattern on a string, within standard formality, a. by stating and proving a theorem expressing the exact resulting intervallic structure mathematically - a set of interval ratios between adjacent terms of a mirrored arithmetic sequence, b. by pursuing the complete mathematical properties of this structure, c. by discussing its various applications, with emphasis put on drillings on flutes, d. by calling upon practice in order to spot actual historical manifestations of the theorem and its discussion, e. by hypothesizing the rôle of the particular conditions implicit in the theorem towards the historical emergence and evolution of two primeval prototypes of the most prominent and universally disseminated intonational systems / "ur-scales": an anhemitonic pentatonic one ([C, $\left.\left.\mathrm{D}, \mathrm{E}^{1 / 4} \uparrow, \mathrm{G}, \mathrm{A}+\right]\right)$ and the lower fifth of a "smooth" heptatonic one ([D, $\mathrm{E} 1 / 4 \downarrow, \mathrm{F}+, \mathrm{G}, \mathrm{A}+])$.

Keywords: String; Aliquot fingering / fretting; Flute / finger-hole; Interval ratio; Musical scale; Arithmetic / harmonic sequence; Harmonic mean.

\section{Перí $\eta \psi \eta:$}

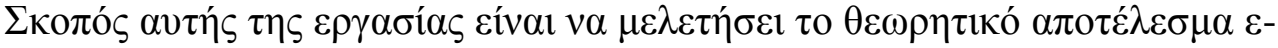

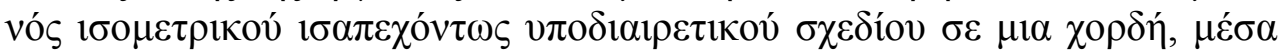

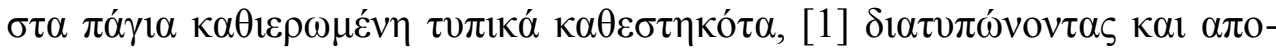

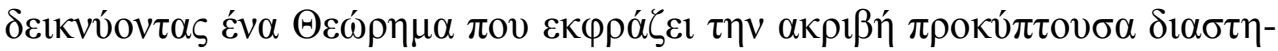

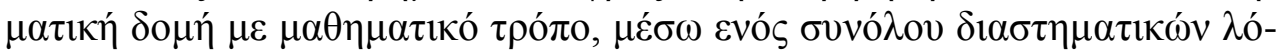

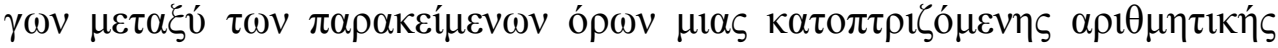

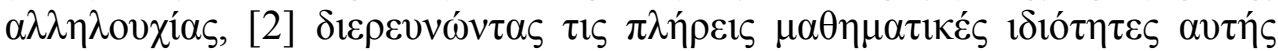

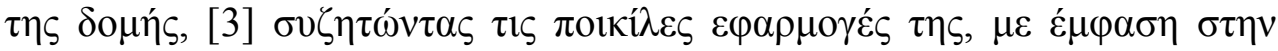

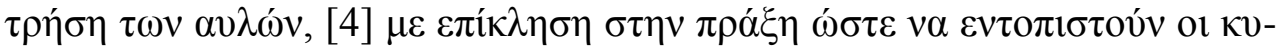

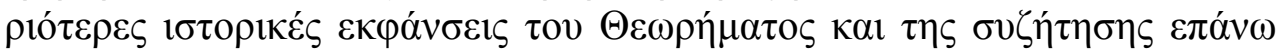

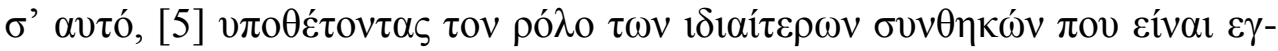




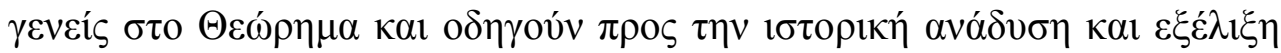

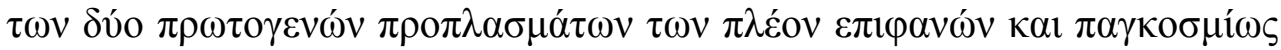

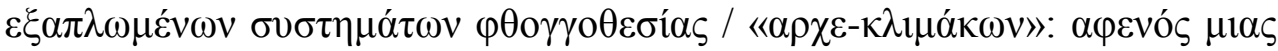

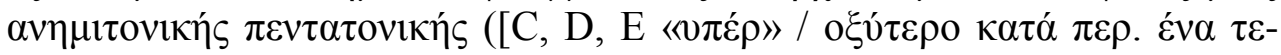

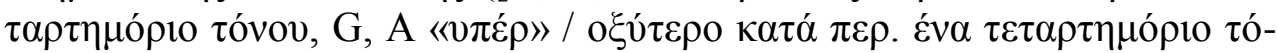

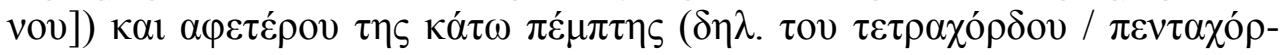

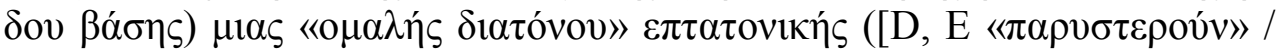

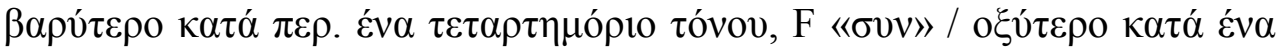

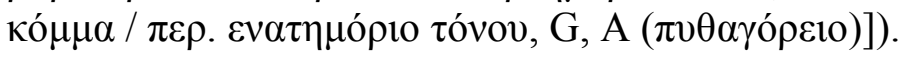

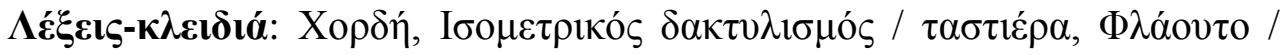

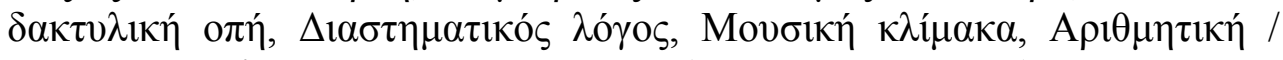

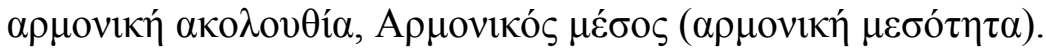

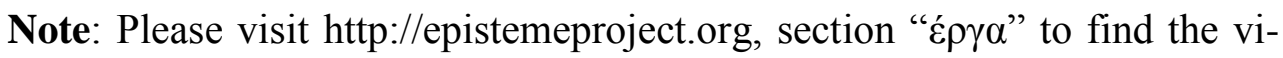
deo where you may listen to various intervals presented in this paper.

\section{Preliminaries}

Let there be a musical string of irrelevant mass and diameter, stretched at an irrelevant tension between points $N$ (nut) and $B$ (bridge), distanced at total length $L$. Let there be a fingering or fret at point $X$, distanced from the nut at a fraction $r$ of overall length $L$ of the string, so length $[N X]$ is $r L$.

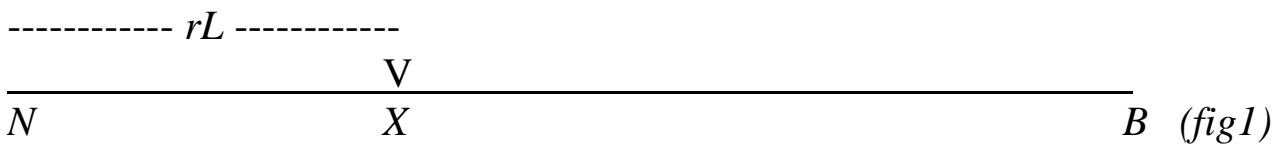

Let the frequency (/ pitch) produced by the total length of the string (note $\mathrm{N})$ be $f_{N}$ and let the frequency (/ pitch) produced by fingering / fretting at point $X$ (note $\mathrm{X}$ ) be $f_{X}$.

Given that length $[X B]$ is $L-r L=(1-r) L$, these two frequencies / pitches are given by the following formulae, for some $c$ serving as a cumulative constant for the string's physical parameters.

$f_{N}=\frac{c}{L} \Rightarrow f_{X}=\frac{c}{(1-r) L}$

The interval ratio $f_{X} / f_{N}$ between the two pitches $X$ and $N$, i.e. the interval by which $f_{X}$ is higher than $f_{N}$, simplifies to $1 /(1-r)$. Let us call that $R$. In other words,

$f_{X} \equiv R f_{N}$ 


$$
R=\frac{1}{1-r} \Leftrightarrow r=R-\frac{1}{R}=\frac{R-1}{R}
$$

Suppose now that we split segment $[N X]$ into $k$ aliquot parts by interposing $(k-1)$ equally spaced fingering points / frets, so that the nut $N$ corresponds to the $0^{\text {th }}$ ordinal point and $X$ to the $k^{\text {th }}$ one; suppose, also, that we name the $j^{\text {th }}$ such point $Y(0 \leq j \leq k)$, so that length [NY] is $j / k$ times length [NX]. Then the following holds:

$$
\begin{aligned}
& {[N Y]=\frac{j}{k}[N X]=\frac{j}{k} r[N B]=\frac{j}{k} r L} \\
& r L \text {------------- } \\
& \begin{array}{l|lll|l|l}
\lfloor\downarrow & \ldots & \mathrm{V} & \ldots & \mathrm{V} \\
\hline N & Y & & X
\end{array}
\end{aligned}
$$$$
---(j / k) r L \text {--- }
$$

In order to determine the frequency $f_{Y}$ of pitch produced at point $Y$ (note $\mathrm{Y}$ ), we observe that the vibrating segment $[Y B]$ of the string fingered or fretted at point $Y$ is

$$
L-\frac{j}{k} r L=\left(1-\frac{j}{k} r\right) L \Rightarrow f_{Y}=c /\left(1-\frac{j}{k} r\right) L
$$

Suppose now we place an extra fingering / fret at point $Z$, so that segment $[Z Y]$ is the $j^{\text {th }}$ aliquot segment of our subdivision. $Z$, then, must be the very preceding point, i.e. the $(j-1)^{\text {st }}$ one. Again we have note $Z$ at some pitch / frequency to be determined.

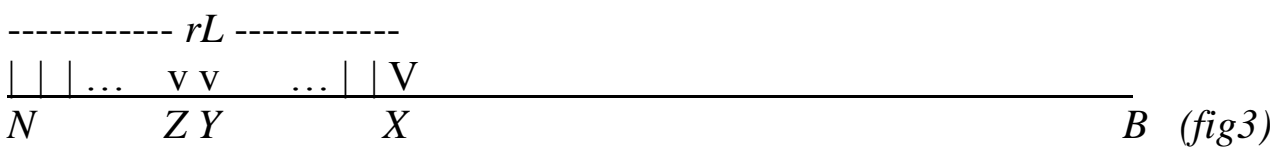

$---(j / k) r L---$

The immediate objectives of our quest are to study a. the intervallic result of the particular fingering / fretting point $Y$ set in place in the particular fashion or, in other words, the tonal relationships (interval ratios) between notes $\mathrm{Y}$ and $\mathrm{N}$ and between notes $\mathrm{X}$ and $\mathrm{Y}, \mathrm{b}$. the rational relationship between two consecutive points of an equitable fingering / fretting subdivision in the specific case of the interval ratio corresponding to the $j^{\text {th }}$ such segment, towards building the general case.

In the process we need keep track of a situation stemming from the tricky enumeration applied: for segments to be counted from $1^{\text {st }}$ to $k^{\text {th }}$, subdividing points must be numbered from $1^{\text {st }}$ to $(k-1)^{\text {st }}$; if we include the endpoints, ordinals go from $0^{\text {th }}$ to $k^{\text {th }}$. 


\section{The theorem}

\subsection{Convention}

All musical intervals mentioned in this paper as frequency quotients are assumed ascending. In this sense, ratios expressing them must have their numerator $\geq$ their denominator; wherever operations produce descending intervals, ratios will be inverted. In order to cope with this situation, we need an extra auxiliary definition.

\subsection{Definition I}

Two interval ratios $a / b$ and $c / d$ are said to be ascending-equivalent iff $\boldsymbol{a} / \boldsymbol{b}$ $=c / d$ or $a / b=d / c$.

\subsection{Lemma}

In an arithmetic subdivision of a given numerical interval $[w, R]$, where $w<$ $R$, into $k$ equal parts:

a. the $m^{\text {th }}$ subdividing point is given by general formula $w+(R-w) \frac{m}{k}$;

b. the $m^{\text {th }}$ ratio between two successive subdividing points is the one between $m^{\text {th }}$ and $(m-1)^{\text {st }}$ point; the general formula for its ascending version is

$\left[w+(R-w) \frac{m}{k}\right] /\left[w+(R-w) \frac{m-1}{k}\right]$.

Proofs follow directly from the definitions.

\subsection{Definition II}

A subdivision of a "reference interval" $R$ complying with the procedure given in the Preliminaries in chapter 1, i.e. as a result of an equitable fingering / fretting scheme, shall be called an "(upwards) harmonic subdivision".

\subsection{Theorem}

The constituent -ascending- intervals produced by an "(upwards) harmonic subdivision" of "reference interval" $R$ into $k$ segments duplicate the results of an arithmetic subdivision (i.e. an aliquot spacing of frequency units / Hertz contained) of the same reference interval, $R$, into the same number of segments, $k$, in the reverse order.

\subsection{Proof}

First let us indeed determine the ascending tonal / intervallic relationship of note $\mathrm{Y}$-placed at the $j^{\text {th }}$ subdividing point- with respect to the endpoints of 
the subdivision, i.e. to notes $\mathrm{N}$ and $\mathrm{X}$; by skipping a couple of intermediate operations and by expressing both in terms of $R$ :

$$
\begin{aligned}
& \frac{f_{Y}}{f_{N}}=1 /\left(1-\frac{j}{k} r\right)=1 /\left(1-r+r-\frac{j}{k} r\right)=R /\left[1+(R-1)\left(1-\frac{j}{k}\right)\right] \\
& \frac{f_{X}}{f_{Y}}=\frac{f_{X} / f_{N}}{f_{Y} / f_{N}}=R /\left\{R /\left[1+(R-1)\left(1-\frac{j}{k}\right)\right]\right\}=1+(R-1)\left(1-\frac{j}{k}\right)
\end{aligned}
$$

Already we know that the straight, simple expression contingent on $j$ is the latter one, which ties note $\mathrm{Y}$ to note $\mathrm{X}$-because it involves a direct proportionality, as it features $j$ at a numerator of its second term-, whereas the former ascending ratio, between $\mathrm{Y}$ and $\mathrm{N}$, carries a complicated inverserelated proportion to $j$-lying at the denominator.

Let us now turn our attention to extra note $\mathrm{Z}$ and its own pitch. By the same token, as it is sounded at the $(j-1)^{\text {st }}$ subdividing point, its ascending equations turn out thus:

$$
\begin{aligned}
& \frac{f_{Z}}{f_{N}}=R /\left[1+(R-1)\left(1-\frac{j-1}{k}\right)\right]=R /\left[1+(R-1) \frac{k-j+1}{k}\right] \\
& \frac{f_{X}}{f_{Z}}=1+(R-1)\left(1-\frac{j-1}{k}\right)=1+(R-1) \frac{k-j+1}{k}
\end{aligned}
$$

As the $j^{\text {th }}$ ascending (upwards) harmonic ratio is that between $j^{\text {th }}$ and $(j-1)^{\text {st }}$ points:

$$
\frac{f_{Y}}{f_{Z}}=\frac{f_{X} / f_{Z}}{f_{X} / f_{Y}}=\left[1+(R-1) \frac{k-j+1}{k}\right] /\left[1+(R-1) \frac{k-j}{k}\right]
$$

By defining $\mu$ as the difference between $k$ and $j$, i.e. between denominator and numerator of the ratio characterizing $\mathrm{Y}$-the point interposed-, $j$ becomes $(k-\mu)$ and

$$
\frac{f_{Y}}{f_{Z}}=\left[1+(R-1) \frac{\mu+1}{k}\right] /\left[1+(R-1) \frac{\mu}{k}\right]
$$

Yet, because of the tricky nature of our ordinal enumeration from 0 rather than from 1 , this would be the $(\mu+1)^{\text {st }}$ ascending interval ratio; the $\mu^{\text {th }}$ one should be:

$$
\left[1+(R-1) \frac{\mu}{k}\right] /\left[1+(R-1) \frac{\mu-1}{k}\right]
$$

First and foremost, this result duplicates exactly the formulae of our lemma, with unity in the place of $w$ and with $\mu$ supplanting $m$. Second of all, it appears to be a. directly related to the top note of the subdivision, b. $\mu$ - 
dependent, i.e. running backwards, as $\mu$ is a difference term and reduces from $k$ to 0 as $j$ grows from 0 to $k$.

\subsection{Comments}

This concludes the proof of the $2^{\text {nd }}$ main question posed by the introduction to the theorem, as it says that the $j^{\text {th }}$ interval ratio corresponding to each pair of consecutive subdivision points, placed at $(j-1)$ and at $j k^{\text {ths }}$, is ascending-equivalent to the $\mu^{\text {th }}$ ratio in an arithmetic subdivision, i.e. that between $(\mu-1)$ and $\mu k^{\text {ths }}$, which is the same thing as that between $(k-j)$ and $[k-(j+1)]$ or, in other words, the one found by counting the ratios backwards or mirrored from last ordinal number to zeroth. This is an extremely important result, as it unveils two essential properties of an equitably spaced fingering / fretting subdivision of a certain reference interval:

- the intervallic subdivision produced yields the same set of ascending results as that of an arithmetic subdivision into $k$ in the reverse / mirrored order (q.e.d.);

- the basic harmonically meaningful set of relations of the subdividing pitches occurs towards a common reference pitch, which lies at the top of the ascending interval being subdivided, unlike an arithmetic subdivision, where the bottom pitch constitutes the tonal, modal and harmonic focus.

\section{Study}

Several music-theoretical issues are settled by this theorem.

\subsection{Flutes}

Ideally, our results are equally valid for holes on cylindrical flutes grosso modo, given one chief difference and a clarification. Difference: $L$ here corresponds to some specific effective length depending on the particular mix of the flute's parameters each time; usually, actual lengths of flutes are visibly shorter than $L$ towards the mouth-hole and longer at the far end, which is why, at end-blown and whistle-type flutes (shakuhachi, kena, recorder), the mouth is customarily made at the same size as finger-holes. Otherwise the proportion is more or less sensitive to a series of factors like relation of hole size to bore diameter, distance of next open hole etc. If, for instance, we assume that a. point $N$ represents the open free end of a flute or a recorder, b. point $X$ corresponds to a finger-hole sized exactly the same as the hole at the free end and somehow tuned to a specific reference interval, 
c. the in-between segment of the pipe is drilled at a number of equally sized and shaped and evenly spaced finger-holes, our results practically apply just as well, regardless of whatever discrepancy between effective and actual lengths. ${ }^{1}$ Clarification: not all holes need necessarily be drilled, in which case all we may have to do is to assume a number of intervening "virtual holes", granted certain versatile theoretical adjustments. ${ }^{2}$

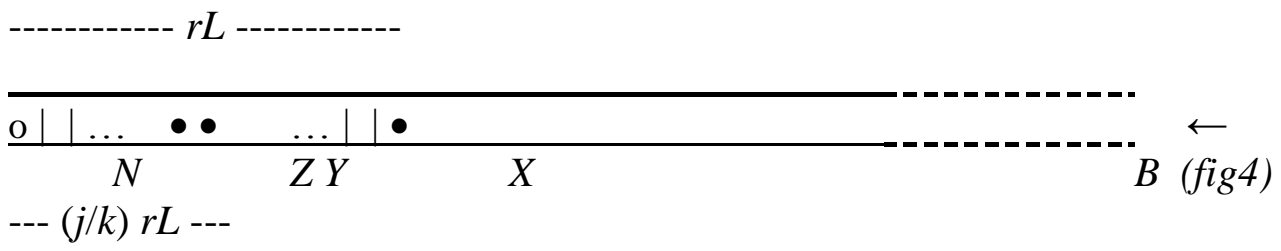

\subsection{Subdividing a superparticular interval}

Let us simplify the analysis for a moment by assuming the simplest case possible, where relative reference pitch $R$ is a superparticular fraction, i.e. of the form $(n+1) / n$. It is vital to note that no loss of generality occurs, because the results are analogous for all other rational fractions, give or take a few cancellations of common factors. Most of the usual crucial divisible reference intervals are superparticular anyhow.

Since our relations work both ways throughout, it is reasonable to make the following observations, assuming that we wish to subdivide the abovementioned reference interval $[(n+1) / n]$ into $k$ parts, and given that we define number $K$ as the product $k n$.

${ }^{1}$ Typically, our interest in flutes is focused on distances from the far end. All an unequally sized open end does is blow the length of the bottom joint out of proportion. The situation can be remedied by simply assuming a different virtual / effective length for that joint only, properly contingent on the overall drilling pattern. The said constriction of the mouth of certain flutes is one way around "faulty" analogy of lengths. The "extreme" width of fingerholes on several archaeological finds of ancient cylindrical auli, at diameters adjusted so as to match the mouth, affords a second standard way.

${ }^{2}$ By the same token, not all fingerings need be fretted under a string; absent "virtual frets" or "extra in-between frets" are conceivable and reported as well. A creative manipulation of this type of considerations and their varieties leads directly to a set of theoretical concepts and controls capable of forming a compact model for dealing with flutes, or even with mere fragments of flutes; in fact, it contributes towards the formulation of a powerful analytical tool useful towards studying the intervallics of just about any 1-dimensional sound source; this model will be the object of a future more specialized paper. 
3.2.1. As is widely known, an arithmetic subdivision of a superparticular reference interval produces an ordered sequence of $k$ successive ascending fractions of the forms

$$
\frac{K+1}{K}, \frac{K+2}{K+1}, \frac{K+3}{K+2} \ldots \ldots \frac{K+k-2}{K+k-3}, \frac{K+k-1}{K+k-2}, \frac{K+k}{K+k-1}
$$

For those concerned, in the particular case under discussion, this is a segment of the complete harmonic series between partials $k n$, or $K$, and $k(n$ $+1)$, or $(K+k)$, of some fundamental, ${ }^{3}$ and its consecutive frequencies define an arithmetic progression. All successive intervals formed in our example are superparticular and their ratios (therefore their perceived "sizes") shrink as we go upwards. On the other hand, all pitches of the constituent note series bear a common tonal relationship to the bottom note, habitually called "the root", so that we can say that the particular succession of fractions is "tonally orientated to the bottom". By Fourier's theorem, this is the only rigorous, theoretically sound and theory-bound subdivision of an interval.

3.2.2. By the theorem we just proved here, an (upwards) harmonic subdivision of a superparticular reference interval produces the following ordered set of successive ascending fractions:

$$
\frac{K+k}{K+k-1}, \frac{K+k-1}{K+k-2}, \frac{K+k-2}{K+k-3} \ldots \ldots \frac{K+3}{K+2}, \frac{K+k-1}{K+k-2}, \frac{K+1}{K}
$$

Again, all successive intervals formed are superparticular, but now their ratios expand as we go upwards. Let us see how this is done by a superparticular numerical example. The interval ratio of a fifth is $3 / 2$, meaning e.g. that the frequency of a $\mathrm{G}$ is 1.5 times the frequency of a $\mathrm{C}$. In order to split a fifth 3 ways arithmetically, we multiply both terms of the ratio by 3 and proceed as follows:

- arithmetic trisection of $2: 3=$ arithmetic trisection of $6: 9=6: 7: 8: 9$;

- ascending arithmetic $3^{\text {rd }}$ parts: $(6: 7=) 7 / 6,(7: 8=) 8 / 7,(8: 9=) 9 / 8$;

- the harmonic trisection is determined by the inverse sequence $9: 8: 7: 6$;

- ascending (upwards) harmonic $3^{\text {rd }}$ parts of the fifth: $9 / 8,8 / 7,7 / 6{ }^{4}$

\footnotetext{
${ }^{3}$ This is only true of some classes of cases, superparticular being one of them. It is not true, for instance, of bounded arithmetic sequence segments $[6,6+(\sqrt{2} / 2), 6+\sqrt{2}]$ or $[3,5,7,9]$, neither one of which is a segment of any complete harmonic series. For more details see footnotes 4 and 22 .

${ }^{4}$ The procedure is also known as catapycnosis. The resulting fractions may need to be simplified by cancellation of common factors. In the general rational case, $R=(n+l) / n$. Let $D=\operatorname{GCD}(k, l)$; then $K$ is defined as $k n / D,(K+k)$ is replaced by $[k(n+l) / D]$ and,
} 
This time all pitches of the constituent note sequence bear a common tonal relationship to the top note; so we can say that the particular succession of fractions is "tonally orientated to the top". Given Fourier's theorem, this is a theoretically unsound subdivision of an interval; it can be safely inferred that it has a direct provenance in theoretically ignorant / naïve musical practice and, whenever it is observed there, it has almost certainly sprung from easily conceived and executed equidistant borings of flutes and, perhaps later, fingerings / frets on strings, unless it be the result of some $\mathrm{ad}$ hoc "mathematical" computation or "principle". Even then, structures developed in this way can clearly be seen as anti-theoretical and extending "upside down".

3.2.3. Indeed, as anyone familiar with but the most rudimentary aspects of Acoustics can readily understand, if what matters is the vibrating part of the string or aerial column, measuring distances and defining their relations from the nut of a string or from the free far end of a flute, and then physically and mentally working on lengths thus obtained, is tantamount to illegitimately correlating silenced parts, thence to doing the job from the wrong end.

However, it is a fact that actual musical systems have a habit of developing in this fashion, and the upwards harmonic subdivision has found its way into theoretical treatises once too often, especially on masses of "accurate theory" derived from studying music on lutes. Besides we all know that primitive and folk flutes -both in the temporal / prehistoric sense and in the controversial cultural context- exhibit a strong tendency to come out thus drilled and it is the author's assessment that at least some of the most prominent and wide-spread musical systems in the world (see VI at end of paper) trace their origins in this very principle of scale- and modegeneration.

finally, instead of 1 , the increment in the upcoming equations is $l / D$. But there is nothing in the theorem presupposing rationals, so the ideas expressed here apply perfectly well in any situation; irrational or transcendental interval quotients are also embraced. For instance, we could arithmetically trisect interval $[1, \pi]$ a. by taking arithmetic subdivision points i. $(2+$ $\pi) / 3$, ii. $(1+2 \pi) / 3$, b. by defining ratios determining the three consecutive arithmetic third parts, i.e. i. $(2+\pi) / 3$, ii. $(1+2 \pi) /(2+\pi)$, iii. $3 \pi /(1+2 \pi)$ and, finally, c. by reversing their order so as to obtain the three consecutive upwards harmonic third parts.

${ }^{5}$ The only case where this principle is rigorously applicable is strict 2-part voicing, where symmetry between "upwards" and "downwards" is implicit. 


\subsection{Practicability of harmonic subdivisions}

The (upwards) harmonic subdivision of an interval is easy to conceive and quite straightforward to implement in the construction and playing of conventional musical instruments; but at the same time it has proved tricky and confusing in its computations. On the contrary, the arithmetic subdivision has shown itself to be impracticable in instrument-building and in music-making -in fact, probably few instruments in the world are drilled or fretted arithmetically-, but its theory is computationally elementary. By employing the present theorem, all one has to do is to perform an arithmetic subdivision and then simply reverse the order of the outcome.

\subsection{Generality of approach}

No loss of generality occurs due to measuring the entire effective rather than actual length for the lower point of our interval, or even due to failing to do so. It is just a matter of convention. The results hold for subdividing an intermediate segment too, if we consider and treat the root of our reference interval as a "temporary" "nut" or "far end of the flute", a fact allowing study of broken flutes with at least three holes. For a complete study, one ought to substitute relevant effective for actual length at the flute's farthest section. ${ }^{6}$

\subsection{The harmonic mean}

The leading application of the principle and procedure in question here has been one of bisecting a given interval, whence the choice of terminology in this paper. In this case, the simplest one of all, $k=2$, and $j$ as well as $\mu$ can only take on intermediate extra value of unity.

Greek theorists used to define three chief intervallic "midpoints", which they used to call "arithmetic", "geometric" and "harmonic" means. By the present theorem, the "harmonic mean" becomes one special instance of a general category of subdivisions that has been incompletely understood. Here, the adjective "harmonic" has been picked up directly from the Greek, and the optional qualifying adverb "upwards" has been applied for clearing out and constantly reminding its particular innate harmonic function with respect to the top note as determined above.

\footnotetext{
${ }^{6}$ What's more, one can also take care of an occasional unequally sized finger-hole in appropriate cases, by applying familiar formulae of Musical Acoustics towards computing a virtual adjusted alternative / substitute positioning of an equivalent "virtual equally sized" finger-hole in lieu of an actual one.
} 
Classical writers gave us the formula for the "harmonic mean", without so much as once convincingly justifying why they would place the much more intricate algebraic expression $2 a b /(a+b)$ in the same basket as the natural ordinary ones for arithmetic and geometric means, i.e. $(a+b) / 2$ and $(a b)^{1 / 2}$ respectively. ${ }^{7}$ There is a shortage of relevant comments in the literature; it is as if they either never realized the fact that here they were talking about something as conspicuous as "halving" by means of a midpoint fingering or bridge or fret or finger-hole -a practice with apparent historical origins in folk music since the very dawn of mankind- or had some reason to disregard or conceal it. Even the explanations they gave for it are at least farfetched. On the other hand, it seems that hosts of scholars commenting in later times have been tending to a. miss the musical nature and generative process of the "harmonic mean", b. fail to perform the algebraic operations in the formula so as to relate it to some physical meaning, c. never ask themselves how and why such a curious or, say, intricate concept ever made its way amidst two other perfectly straightforward ones, occasionally driven to think that there may be a mystical significance behind it, or, much later, fascinated by the lovely mathematical properties and exciting applications of "harmonic sequences" and means in statistics and in modern mechanics.

In fact, soon several of this mean's features were perceived, as, for instance, $i$. that the product of arithmetic and harmonic mean equals the square of the geometric mean, ii. that the geometric mean of two numbers is the same as the geometric mean of their arithmetic and harmonic means, the combination of these two thus affording a very clever and quickly convergent way for calculating square roots. ${ }^{8}$ Yet other things have been long missed, such as the potential of defining classes of multiple harmonic subdivisions / sequences way beyond "halves", or the plausible likelihood that the very idea had originated in musical practice tens of millennia into the past, quite possibly on Paleolithic flutes such as Cro-Magnon ones found in the Madeleine caves at Dordogne in France and dated between 1 and 4 tens of millennia b.C. Slight variations of the same concept are visually conspicuous on a 9000-year-old batch of flutes unearthed in Central China not long ago.

${ }^{7}$ The term harmonicos is first mentioned in a surviving fragment by Archytas ( $5^{\text {th }}$ c. b.C.), as a synonym to (mesos) hypenantios (= subcontrary). There is an example of an involved and rather confusing explanation of its nature in Nicomachus Gerasenus's (2 ${ }^{\text {nd }} c$. a.D.) Arithmètikè eisagōgē II.25; see bibl.

${ }^{8}$ Some alternative equations: a. $\mathrm{AM} \times \mathrm{HM}=\mathrm{GM}^{2}$, b. $\mathrm{HM}=\mathrm{GM}^{2} / \mathrm{AM}, \mathrm{c} . \mathrm{GM}=(\mathrm{AM} \times \mathrm{HM})^{1 / 2}$. Iterations quickly narrow down the interval inside which the geometric mean sits. 


\section{Historical examples}

Harmonic subdivisions are to be found in the writings of several theorists in classical and medieval times. Two notable modern scholars are also cited.

\subsection{Plato}

The harmonic mean is a fundamental concept in the proportions of the (material) cosmic psyche in Plato's (427?-347 b.C.) Timoeus, ${ }^{9}$ a dialogue essentially laying out Pythagorean doctrine in contexts far surpassing plain arithmetic, music or geometry.

\subsection{Eratosthenes}

The chromatic pycnon of Eratosthenes (275-194 b.C.) ${ }^{10}, 20 / 19$ and 19/18 -a structure duplicated by ibn Sînâ or Avicenna (980-1037) in his own genus VIII (chromatic chroa 1$)-{ }^{11}$ is a harmonic bisection of a grave tone (10/9) into two half-tones; his enharmonic pycnon, 40/39 and 39/38, is a harmonic bisection of his lower half-tone. The two subdivisions put together can be said to form a partial / elliptical harmonic quadrisection of the grave tone, with the third point omitted $(40 / 39,39 / 38,38 / 36)$.

\subsection{Didymus}

The diatonic ditone / just major third of Didymus (63 b.C.-10 a.D.) is harmonically bisected into grave and epogdoos tones, 10/9 and 9/8 -a practice adopted by ibn Sînâ (genus V, diatonic chroa 5) and, much later, also by Rameau (1683-1764)-; ${ }^{12}$ the enharmonic pycnon of the same theorist, $32 / 31$ and $31 / 30$, is a harmonic bisection of a just semitone (16/15); however ibn Sînâ in his own genus XII (enharmonic chroa 1) unreverses the order of the quarter-tones (31/30 and 32/31), establishing an arithmetic bisection. A Didymus-Rameau type of just diatonic "lydian" or "major" tetrachord $[\mathrm{C}, \mathrm{D}-, \mathrm{E}, \mathrm{F}]^{13}$ is attainable by an elliptical harmonic quintisection: by equitably splitting the space of a fourth 5 ways and omitting the $1^{\text {st }}$ and $3^{\text {rd }}$ ordinal points (i.e. taking the $0^{\text {th }}, 2^{\text {nd }}, 4^{\text {th }}$ and $5^{\text {th }}$ ones, $c f$. A.4.3 in App. A). ${ }^{14}$

\footnotetext{
${ }^{9}$ In Plato chapters Z (VII) and H (VIII).

${ }^{10}$ For the ratios of Eratosthenes and of Didymus, further on, see Ptolemy's Harmonics 2.14, in Barker: 346-349. For Ptolemy's chroce see ibid: 1.14 and 1.15: $305 \mathrm{ff}$.

${ }^{11}$ For this and all other citations of ibn Sînâ see his pp. 145 ff. See also Jedrzejewski.

${ }^{12}$ Rameau, example I.7: 28.

${ }^{13}$ Actually: the "relative major" / median mode of the just minor scale.

${ }^{14}$ This is noted as "the birth of our major scale" in Schlesinger: 52; $c f$. footnote 2. Long folk flutes in the Balkans and in Anatolia constitute a special category (kaval et al.) drilled
} 


\subsection{Ptolemy}

Ptolemy's ( $2^{\text {nd }}$ c. a.D.) most striking and celebrated chroa, the homalon diatonon (= smooth diatonic), is a genuine harmonic trisection of the perfect fourth (4/3) into $12 / 11,11 / 10$ and 10/9, extant from time immemorial (though we are never explicitly told so), also to be found in ibn Sînâ (genus VII, diatonic chroa 7). It should not be missed that this structure can be extended upwards as a quadrisection of the perfect fifth by just keeping the three intervals in place and topping with an extra (epogdoos) tone of 9/8 (cf. A.2.3 and A.3.2 in App. A).

\section{5. ibn Sînâ / Avicenna}

In addition to these, ibn Sînâ, in the "ditone" of his genus II (diatonic chroa 2), furnishes an example of a supermajor third (9/7) harmonically bisected into $9 / 8$ and $8 / 7$-tone and supertone. ${ }^{15}$ The structure goes [28/27, 9/8, 8/7] or $[\lambda L, T, T 7]$ and the corresponding notes from $\mathrm{C}$ are $[\mathrm{C}, \mathrm{Dk}-, \mathrm{Ek}, \mathrm{F}]{ }^{16}$

\subsection{Eccentric splits}

Ptolemy has also coined a particular brand of eccentric splits, which are in fact disguised elliptical trisections -with the $2^{\text {nd }}$ dividing point omitted. His chroce are filled with arithmetic instances of this idea. Yet there is also a harmonic one in his unique soft "diatonic" [21/20, 10/9, 8/7] or [SL, t, T7], where the two lower steps can be rewritten as 21/20, 20/18. The structure is duplicated by ibn Sînâ as his genus III (diatonic chroa 3) (notes from C: [C, $\mathrm{D} k, \mathrm{E} k, \mathrm{~F}])$.

\subsection{Just / Zarlino minor chords}

It should not be neglected that, as the above table shows, just minor triadic and tetradic chords (e.g. in Zarlino's tuning, a.D. 1562) are a harmonic bisection of the perfect fifth and a harmonic trisection of the octave respectively, thus giving minor just harmony a character of harmonic subdivisions and showing just minor chords to be upwards-orientated and

along this principle but in full chromatic catapycnosis, i.e. with all these six holes as in App. A: A.4.3 actually present (plus some more).

15 All in all, ibn Sînâ's structures contain i. seven means (three arithmetic, one geometric and three harmonic), ii. one harmonic trisection.

${ }^{16}$ It can also be viewed as an elliptical septisection: 28/27, 27/24, 24/21. Both Archytas and

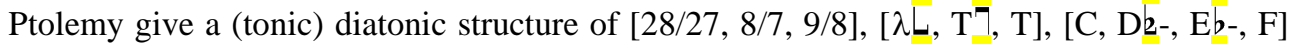
instead, with the upper part halved arithmetically rather than harmonically. For Archytas's ratios see Ptolemy's Harm. 1.13 in Barker: 43-52. 
thus, effectively, upside down. In contrast, the arithmetic trisection of the octave is a just major chord in second inversion (App. A: A.2.1).

\subsection{Prolemy's homalon diatonon}

The property cited above at example 3, regarding Ptolemy's homalon diatonon, is an instance of the fact that an (upwards) harmonic subdivision can be extended past either edge. The progressive expansion effect makes the products of the subdivision, i.e. its steps, differ greatly in width from end to end when the subdivided reference interval is wide, say of the order of the octave.

\subsection{Boethius}

Roman music theorist Boethius (480-524 a.D.) used a method based on string lengths quite extensively. Harmonic divisions come in abundance in his work, yielding series of ratios of whole numbers that can be deemed nothing short of peculiar. Here is an account of his method and results, as worked out and tabulated by Joe Monzo:

- between E+ and "Gb": [512/499, 499/486, 486/473, 473/460] (increment: 13);

- between "Gb" and "F\#": [460/459, 459/458];

- between "F\#" and G: [458/445, 445/432] (increment: 13). ${ }^{17}$

\subsection{Fârâbî}

Fârâbî (878-951) has been the recipient of major influences from ibn Sînâ. In his famous treatise Kitâb al-mûsîqî al-kabir, this celebrated medieval scholar gives a list of 13 mathematically expressed "Greek" genera. In it he includes a number of Greek-based tetrachords that are all more or less mirrored (i.e. turned upside down), among some others which he himself has devised by way of mathematical considerations. All of them are put down as string lengths in the frettings of model lutes, thus spanning large portions of his integrated modal system.

Fârâbî distinguishes his "Greek" genera into two categories: a. soft, ordered (meaning ones with pycnon, i.e. enharmonic and chromatic indiscriminately) and b. strong (i.e. diatonic). In the "Greek" tetrachords Fârâbî lists, as compared to the genuine ones, some (upwards) harmonic partitions turn out arithmetic and vice versa, though not all. Among the former, one finds five harmonic divisions; two of these are true ("continuous") bisections, cited below in J.P.N. Land's Latinized nomenclature -the other

\footnotetext{
${ }^{17}$ Boethius R.15.22 (944), folios 48v-65v. Also see Monzo.
} 
three are along the line of Ptolemy's (“discontinuous") eccentric splits / elliptical trisections: ${ }^{18}$

Genus molle, ordinatum / continuum :-

a. laxum: [5/4, 32/31, 31/30], [III, $\Delta \mathrm{q}, \mathrm{Sp}]$, [C, E, E\#q, F], a quasi-reversal of Didymus's enharmonic;

b. mediocre: $[6 / 5,20 / 19,19 / 18]$, [iii, $\Delta \varphi, \mathrm{S} \gamma],[\mathrm{C}, \mathrm{Eb}, \mathrm{E} \varphi, \mathrm{F}]$, a quasireversal of Eratosthenes's chromatic. ${ }^{19}$

From the scope of this paper, though, the most striking feature in Fârâbî's writings is indeed his analysis of flutes and their finger-holes; his basic approach concentrates on an aliquot drilling and is essentially very close to ours. $^{20}$

\subsection{Pachymeres}

Byzantine scholar Georgius Pachymeres (1242-c. 1310) repeats all of Ptolemy's chroce plus adds three other possibilities of dividing a tetrachord into three superparticular steps, but states that they are unused by musicians. ${ }^{21}$ Two of these, as they would be laid out in a sense "epi to oxy" (i.e. going up), both contain harmonic bisections: i. [16/15, 15/14, 7/6], [S, $\Delta 7$, iiil], [C, Db, D 7, F] and ii. [14/13, 13/12, 8/7], [TLu, Tn, T7], [C, DLu, $\mathrm{Ek}, \mathrm{F}]$; the former is a mirroring of al Fârâbî's genus molle, ordinatum, a. continuum, acre, while both are similar to ibn Sînâ's genera IV and V (diatonic chroce 4 and 5), with harmonic bisections in lieu of arithmetic ones. $^{22}$

\subsection{Schlesinger}

Kathleen Schlesinger (1862-1953) is a modern scholar meriting a special citation of some length. Her imaginative work is discredited by many. Her basic idea, largely inspired by the study of primitive flutes and of Ptolemy's homalon diatonon, was that the Greek pitch system has its source in original equidistant and equal-sized prototypes of flute / aulos drillings, covering entire octave spans. This is not the place for a detailed critique of her theses, or for showing why her analytical results could not possibly be a

\footnotetext{
${ }^{18}$ In Helmholtz-Ellis app. xx, section K, II: table of non-harmonic scales: 514-515.

${ }^{19}$ All in all, Fârâbî's list contains i. eight means (three arithmetic, three geometric and two harmonic), ii. four eccentric splits / disguised elliptical trisections (one arithmetic, the rest harmonic), iii. one full arithmetic trisection.

${ }^{20}$ al-Fârâbî: $261 \mathrm{ff}$.

${ }^{21}$ Pachymeres folio 52v; see Tannery: 111.

22 al-Fârâbî ibid; also see Helmholtz-Ellis ibid: 515, tetrachord I.a.3 (\#17); cf. section 4.4 above, as well as footnotes 10 and 11 .
} 
fair actual account of what we know as the classical Greek musical system. For our purposes it is sufficient to start thus: Schlesinger was absolutely correct in her principle, but she lost perspective in applying it over the edge.

More specifically, she was perfectly aware of the narrow results of the theorem in this paper. She focused not on string fingerings but on aulos drillings. She elaborated to the point of writing how an equidistant drilling is generated by a "natural instinct of man" ( $c f .3 .3$ above) and how it produces the "reversal" of a segment of the harmonic series, which she called a "modal series", ${ }^{23}$ much as this again is not true of all classes of cases. $^{24}$

The main pragmatic criticism exercised in the literature against her views is that her assumption about this system is contradicted by archaeological evidence of prehistoric and Greek flutes / auli found. ${ }^{25}$ This line of criticism can be dismissed, on account of

a. the Cro-Magnon and many other prehistoric flutes, such as the nearly isometric batch from China;

b. the fact that all heptatonic Greek and Roman flutes found so far are sophisticated products of city culture, their intonation having already undergone advanced Pythagoreanizing changes for conforming to official practice of the times;

c. Aristoxenus's assertion that, in Athens of his time, a parallel classical auletic school of music was teaching on the basis of a scale with 2 intervals of $3 / 4$ of a tone and 1 of a tone at the bottom as seen on the drilling of auli, ${ }^{26}$ a structure rooted in the results at App. A: A.7.2 and directly pointing towards chapter 5 below.

d. considerations mentioned or hinted at in this paper, ${ }^{27}$ broadening the scope by introducing more general concepts, such as virtual dividing points / elliptical spacing; for instance, such methodology is applicable to the age-old Islamic nay, or to unpublished neolithic flutes recently excavated in Western Macedonia Greece.

Thus, despite lacking hard archaeological evidence of Greek auli with equally spaced holes, if we are to trust Aristoxenus as a creditable literary

\footnotetext{
${ }^{23}$ Schlesinger: 40 and 4.

${ }^{24}$ It is not true if the corresponding arithmetic progression is not a segment of the harmonic series ( $c f$. footnote 3 ) or -which is the same- if the increment is not 1 . This would occur either because of an irrational interval subdivided, or of $l$ being an integer multiple of $D$ other than its own power ( $c f$. footnote 4 ).

${ }^{25}$ See for example West: $96-97$.

${ }^{26}$ Aristoxenus II.26 ff: 108.

${ }^{27} \mathrm{Cf}$. footnote 2 .
} 
source of evidence at all, we probably must accept that such auli existed and were conceivably widespread, on the merit of this passage, much as he himself frowns on the idea.

Schlesinger's work never assumed the format of theorem, proof and study; thus a lot of important side issues stemming from mathematical formality and present in this paper never came up in her texts. On the other hand, regarding over-application in her derivation, computation and/or description of the Greek system, it is the author's conviction that her logic works, as long as one confines oneself a. to the older auletic system, obviously parental to the (syntonic) Greek one, yet still extant in parallel to it throughout the classical era, b. to one-hand diatonic flutes and auli, spanning a range considerably smaller than an octave.

Two relevant remarks are due. First, an one-hand flute can span an octave if it is short and the scale is pentatonic, thus her method could have been directly applicable there, were it not for the distortion cited above in 4.8. ${ }^{28}$ Second, in heptatonic environments, an one-hand flute spans a fourth, a fifth or a sixth. Third, the generalized ambiguity of drillings and scales in the segment above the fifth, which stretches from an incomplete intermediate stage of hexatones to an uneasy variety of intervallic compositions observed in folk flutes, combined with the given conjunct disjunct structure of the classical Greek, Byzantine and Islamic systems, shows that the un-ambiguity is restricted in the lower fifth and in its extensions downward, and suggests an independent stage of drilling above it. Therefore Schlesinger's heptatonic approach ought to have narrowed itself down to a model nucleus of a fourth / fifth, rather than being stretched out to a heptatonic octave, an over-application that gave birth to her familiar controversial, contested and unrealistic results, exciting as they may be.

Indeed, a criticism Schlesinger has repeatedly received is that her division of the octave is arbitrary and extravagant and that, being unsupported by evidence, it is a game of her mind and sounds wrong. The author is willing to partially accept this criticism, within the proper scope though. There is indeed truth in all such theoretical objections put forth, yet they only modify and correct parts of her analysis and her conclusions, without annulling her entire basic idea. Had she applied the exact same principle to the octave not in one but in two pieces -in a context amply

\footnotetext{
${ }^{28}$ She does indeed try to make up by omitting (/ "virtualizing") one or more notes produced in most of her "modal series". Apart from the fact that exceptions are mathematically unacceptable, her method relativizes and pulverizes her very basic assumption of applying aliquot drilling throughout the octave.
} 
supported by ethnomusicological research and in the way this paper is doing in its next chapter (5) -, and had she refrained from speaking of the integral Greek system as it is known, her results would have been both credible and accredited by abundant evidence.

\subsection{Sachs}

The fact of the early emergence of musical systems by aliquot partitions of effectively 1-dimensional sound sources could not pass and indeed has not passed unnoticed by musicology. It is a staple approach in the pioneering work of Curt Sachs (1881-1959), who also ventured to investigate the said emergence from an "equipartition" of flutes and made express note of this particular property as found in Ptolemy's homalon. ${ }^{29}$

\section{Historical prototypes of musical (ur-)scales}

To compensate for the distortion brought about by a large aliquot division (say an octave-wide one), as mentioned in section 4.8, a certain practice can be applied and has indeed been implemented in many cultures throughout history: the reference interval can be split into two conjunct pieces and then both can be subdivided separately. The most prominent and relevant practice is to carry out two harmonic subdivisions applied to a conjunct compilation of a perfect fourth on top of a perfect fifth (or the other way around). Apparently this practice, the only one in perfect compatibility with overblown flutes and with structures involving conjunct - disjunct tetrachords, has given mankind two historic scales / modes of conceivable great prominence.

a. A fifth split three ways topped by a fourth split two ways: ${ }^{30}$

$[9 / 8,8 / 7,7 / 6][8 / 7,7 / 6]$

[T, T7, iiiL] [T 7, iiiL]

[tone, supertone, subminor third] [supertone, subminor third]

$\left.[\mathbf{C}, \mathrm{D}, \mathrm{E}\rceil, \underline{\mathbf{G}}, \mathrm{A} 7, \mathbf{C}^{\prime}\right]$, or $\left[\mathbf{F}, \mathrm{G}, \mathrm{A} 7, \underline{\mathbf{C}^{\prime}}, \mathrm{D}^{\prime}, \mathbf{F}^{\prime}\right]$

This mode could have served as the archetypal scale for all primary pentatonic modes, as well as for something else: for an alternative viewing as a system of two identical conjunct elliptical tetrachords (trichords of a fourth) atop a proslambanomenos tone, with the tonal centre at the proslambanomenos, thus:

$[9 / 8[8 / 7,7 / 6]][8 / 7,7 / 6]$, or [T [T7, iiiL]] [T7, iiiL]

\footnotetext{
${ }^{29}$ Sachs: 75 and 213.

${ }^{30}$ See A.2.2 and A.1.3 in Appendix A.
} 
Our familiar anhemitonic pentatones seem to be octave species of a slight tonal modification thereof, a fact visually conspicuous on the drilling of a shakuhachi:

[9/8 [9/8, 32/27]] [9/8, 32/27]

$[\mathrm{T}[\mathrm{T}, \chi]][\mathrm{T}, \chi]$

[[tone [tone, trihemitone]] [tone, trihemitone]

$\left[\mathbf{C}, \mathbf{D}, \mathrm{E}+, \underline{\mathbf{G}}, \mathrm{A}+, \mathbf{C}^{\prime}\right]$, or $\left[\mathbf{F}, \mathbf{G}, \mathrm{A}+, \underline{\mathbf{C}^{\prime}}, \mathrm{D}^{\prime}, \mathbf{F}^{\prime}\right]$

b. A fifth split four ways topped by a fourth split three ways: ${ }^{31}$

$[12 / 11,11 / 10,10 / 9,9 / 8][12 / 11,11 / 10,10 / 9]$

[Tw, Tm, t, T] [Tw, Tm, t]

tones: [[ephendecatos or "elassōn", grave epidecatos or "elachistos", epogdoos or "meizōn”] epogdoos or "meizōn”] [ephendecatos or "elassōn", grave epidecatos or "elachistos", epogdoos or "meizōn"] $\left[\mathbf{D}, \mathrm{Ew}, \mathrm{F}+, \mathrm{G}, \underline{\mathbf{A}+}, \mathrm{Bw}+, \mathrm{C}^{\prime}, \mathbf{D}^{\prime}\right]$, or $\left[\mathbf{C}, \mathrm{Dw}, \mathrm{Eb}, \mathrm{F}, \underline{\mathbf{G}}, \mathrm{Aw}, \mathrm{B} b, \mathbf{C}^{\prime}\right]$

This one has almost certainly served as the archetypal / prototypal scale for all primary heptatonic modes. It exhibits the interesting feature seen in section 4.4: its lower tetrachord also forms a perfect fourth split three ways, so the entire formation can be alternatively viewed and treated as a disjunction of two homalon diatonon tetrachords. It is found on hosts of primitive and folk heptatonic or semi-heptatonic flutes and reeds throughout the globe in its true form. A minute tonal modification thereof, also found on many folk flutes, especially round the Eastern Mediterranean, as well as on the Baroque recorder itself, ${ }^{32}$ seems to constitute the direct foundation of both Middle-Eastern and Byzantine musical tonal systems, and is literally cited as such by Zalzal $\left(8^{\text {th }}-9^{\text {th }}\right.$ c. $)$, Fârâbî and Chrysanthus of Madytus $(1770 ?-1846):^{33}$

[[12/11, 88/81, 9/8] 9/8] [12/11, 88/81, 9/8]

$[[\mathrm{Tw}, \mathrm{tm}, \mathrm{T}] \mathrm{T}][\mathrm{Tw}, \mathrm{tm}, \mathrm{T}]$

\footnotetext{
${ }^{31}$ See A.3.2 and A.2.3 in Appendix A.

32 This variation lowers just one of the homalon notes by a Didymean comma and is attained by drilling the corresponding equidistant hole a bit smaller. This fact does indicate that, in this particular instance, the story told by the positioning of holes may be different from that told by their sizes (i.e. original tuning vs. subsequent adjustments). Thus, multiparameter acoustical formulae can be applica-ble in stages. There are analytical accounts of this procedure in Lekkas 2003 and 2006. By the hard core of mathematical intuition, where there be one instance of something there shall be others.

${ }^{33}$ See i. al-Fârâbî: 105 ff and tables at 254 ff, ii. Chrysanthus: 28.
} 
tones: [[ephendecatos or "elasson", grave epidecatos or "elachistos", epogdoos or "meizon"] epogdoos or "meizon"] [ephendecatos or "elasson", grave epidecatos or "elachistos", epogdoos or "meizon"]

[D, Ew, F, G, $\left.\underline{\mathbf{A}+}, \mathrm{Bw}+, \mathrm{C}^{\prime}, \mathbf{D}^{\prime}\right]$, or $\left[\mathbf{C}, \mathrm{Dw}, \mathrm{Eb}-\mathbf{F}, \underline{\mathbf{G}} \mathrm{Aw}, \mathrm{B} b-, \mathbf{C}^{\prime}\right]$

This is the very historic scale affording a systemically accountable and faithful succession of step sizes very nearly of $[3 / 4,3 / 4,1 / / 1 / / 3 / 4,3 / 4,1]$ tones (cf. 4.12.4 above). Here are, then, the names given precisely to the former set of musical notes, conventionally on root $\mathrm{D}$, in the current nomenclature employed in Byzantine chant, constituting its basic nominal "soft" diatonic scale:

$\left[\Pi \mathbf{A}, \beta o v, \gamma \alpha, \Delta \mathbf{I}, \underline{\mathbf{K E}}, \zeta \omega, v \eta^{\prime}, \Pi \mathbf{A}^{\prime}\right]^{34}$

Appendix A: Intervallic results of several key arithmetic and (upwards) harmonic subdivisions

\section{A.1. Bisections}

$k=2$ : arithmetic and (upwards) harmonic means
A.1.1. the octave (VIII, $R=2 / 1, r=1 / 2$ )
arithmetic "halves"
(upwards) harmonic "halves"
$3 / 2,4 / 3$
$\mathrm{V}, \mathrm{IV}$
$4 / 3,3 / 2$
IV, V
fifth, fourth
C, G, C'
fourth, fifth
C, F, C'

A.1.2. the perfect fifth $(\mathrm{V}, R=3 / 2, r=1 / 3)$

arithmetic "halves"

$5 / 4,6 / 5$

III, iii

2 thirds: major, minor

C, E, G

(upwards) harmonic "halves"

$6 / 5,5 / 4$

iii, III

2 thirds: minor, major

$\mathrm{C}, \mathrm{Eb}, \mathrm{G}$

A.1.3. the perfect fourth (IV, $R=4 / 3, r=1 / 4$ )

arithmetic "halves"

$7 / 6,8 / 7$

iiiL, T7

subminor third, supertone

$\mathrm{C}, \mathrm{Ek}, \mathrm{F}$

(upwards) harmonic "halves"

$8 / 7,7 / 6$

$\mathrm{T} 7$, iiiL

supertone, subminor third

C, D 7, F

A.1.4. the major third (III, $R=5 / 4, r=1 / 5$ )

arithmetic "halves"

9/8, 10/9

(upwards) harmonic "halves"

$10 / 9,9 / 8$

$\mathrm{T}, \mathrm{t}$

2 tones: epogdoos and grave (epenatos)

epogdoos

$\mathrm{C}, \mathrm{D}, \mathrm{E}$

$\mathrm{t}, \mathrm{T}$

2 tones: grave (epenatos) and

C, D-, E

\footnotetext{
${ }^{34}$ See Lekkas 2006.
} 
A.1.5. the minor third (iii, $R=6 / 5, r=1 / 6$ ) arithmetic "halves"

(upwards) harmonic "halves"

$11 / 10,12 / 11$

Tm, Tw

2 tones: epidecatos and ephendecatos

$\mathrm{C}, \mathrm{Dm}, \mathrm{Eb}$

$12 / 11,11 / 10$

Tw, Tm

2 tones: ephendecatos and epidecatos

$\mathrm{C}, \mathrm{Dw}, \mathrm{Eb}$

A.1.6. the supertone (or ephebdomos tone) (T7, $R=8 / 7, r=1 / 8$ ) arithmetic "halves"

$15 / 14,16 / 15$

(upwards) harmonic "halves"

$\Delta 7, \mathrm{~S}$

superdiesis, just semitone

$16 / 15,15 / 14$

$\mathrm{C}, \mathrm{C} 7, \mathrm{D} 7$

$\mathrm{S}, \Delta 7$

A.1.7. the (epogdoos) tone (T, $R=9 / 8, r=1 / 9$ )

arithmetic "halves"

$17 / 16,18 / 17$

just semitone, superdiesis

$\mathrm{C}, \mathrm{Db}, \mathrm{D} 7$

$\mathrm{S} \xi, \Delta \psi$

larger and smaller Quintilianian half-tones

(upwards) harmonic "halves"

$18 / 17,17 / 16$

tones

$\mathrm{C}, \mathrm{D} b \xi, \mathrm{D}$

$\Delta \psi, \mathrm{S} \xi$

smaller and larger Quintilianian half-

A.1.8. the grave (or epenatos) tone (t, $R=10 / 9, r=1 / 10$ ) arithmetic "halves"

19/18, 20/19

$\mathrm{S} \gamma, \Delta \varphi$

larger and smaller Eratosthenic half-tones

$\mathrm{C}, \mathrm{C} \sharp \Psi, \mathrm{D}$ tones

$\mathrm{C}, \mathrm{D} b \gamma, \mathrm{D}-$

A.1.9. the just semitone $(\mathrm{S}, R=16 / 15, r=1 / 16)$ arithmetic "halves"

(upwards) harmonic "halves"

20/19, 19/18

$\Delta \varphi, S \gamma$

smaller and larger Eratosthenic half-

31/30, 32/31

$\mathrm{C}, \mathrm{C} \sharp \varphi, \mathrm{D}-$

$\mathrm{Sp}, \Delta \mathrm{q}$

larger and smaller Didymean quartertones

quartertones

$\mathrm{C}, \mathrm{D} b \mathrm{p}, \mathrm{D} b$

(upwards) harmonic "halves"

32/31, 31/30

$\Delta \mathrm{q}, \mathrm{Sp}$

smaller and larger Didymean

$\mathrm{C}, \mathrm{C} \sharp \mathrm{q}, \mathrm{Db}$

\section{A.2. Trisections}

$k=3$ : arithmetic and (upwards) harmonic "third parts"

A.2.1. the octave (VIII, $R=2 / 1, r=1 / 2$ )

arithmetic "third parts"

$4 / 3,5 / 4,6 / 5$

(upwards) harmonic "third parts"

IV, III, iii

$6 / 5,5 / 4,4 / 3$

fourth, major and minor thirds

iii, III, IV

C, F, A, C'

minor and major thirds, fourth

A.2.2. the perfect fifth $(\mathrm{V}, R=3 / 2, r=1 / 3)$

arithmetic "third parts"

$7 / 6,8 / 7,9 / 8$

$\mathrm{C}, \mathrm{Eb}, \mathrm{G}, \mathrm{C}^{\prime}$

iiiL, T7, T

(upwards) harmonic "third parts"

9/8, 8/7, 7/6

T, T7, iiiL

subminor third, supertone, tone

tone, supertone, subminor third 
C, Ek, F, G

A.2.3. the perfect fourth (IV, $R=4 / 3, r=1 / 4$ ) arithmetic "third parts"

$10 / 9,11 / 10,12 / 11$

t, Tm, Tw

3 tones: grave, epidecatos, ephendecatos grave

C, D-, Em, F

A.2.4. the major third (III, $R=5 / 4, r=1 / 5$ ) arithmetic "third parts"

$13 / 12,14 / 13,15 / 14$

Tn, TLu, $\Delta 7$

2 Avicennean spondeia, superdiesis

C, Dn, Ek, E

A.2.5. the minor third (iii, $R=6 / 5, r=1 / 6$ )

arithmetic "third parts"

$16 / 15,17 / 16,18 / 17$

$\mathrm{S}, \mathrm{S} \xi, \Delta \psi$

just semitone, 2 Aristidean half-tones

$\mathrm{C}, \mathrm{D} b, \mathrm{~Eb} b \xi, \mathrm{Eb}$

A.2.6. the (epogdoos) tone ( $\mathrm{T}, R=9 / 8, r=1 / 9$ )

arithmetic "third parts"

25/24, 26/25, 27/26

$\delta, \mathrm{Sn}, \Delta \mathrm{u}$

3 "chromatic dieses" (first one: just)

$\mathrm{C}, \mathrm{C} \sharp-, \mathrm{Dn}, \mathrm{D}$

\section{C.7. the grave}

arithmetic "third parts"

28/27, 29/28, 30/29

$\lambda \mathrm{L}, \mathrm{I} 7 \varepsilon, \Delta \mathrm{k}$

sublimma, 2 "hemiolic dieses"

$\mathrm{C}, \mathrm{D} b-, \mathrm{D} b \varepsilon_{-}^{-}, \mathrm{D}-$
C, D, E7, G

(upwards) harmonic "third parts"

$12 / 11,11 / 10,10 / 9$

Tw, Tm, $\mathrm{t}$

3 tones: ephendecatos, epidecatos,

C, Dw, Eb, F

(upwards) harmonic "third parts"

$15 / 14,14 / 13,13 / 12$

$\Delta 7, \mathrm{TLu}, \mathrm{Tn}$

superdiesis, 2 Avicennean spondeia

$\mathrm{C}, \mathrm{C} \sharp, \mathrm{D} \sharp \mathrm{u}, \mathrm{E}$

(upwards) harmonic "third parts"

18/17, 17/16, 16/15

$\Delta \psi, \mathrm{S} \xi, \mathrm{S}$

2 Aristidean half-tones, just semitone

$\mathrm{C}, \mathrm{C} \sharp \psi, \mathrm{D}, \mathrm{Eb}$

(upwards) harmonic "third parts"

27/26, 26/25, 25/24

$\Delta \mathrm{u}, \mathrm{Sn}, \delta$

3 "chromatic dieses" (last one: just)

$\mathrm{C}, \mathrm{CH}, \mathrm{D} b+, \mathrm{D}$

\section{A.3. Quadrisections}

$k=4$ : arithmetic and (upwards) harmonic "quarters"

A.3.1. the octave (VIII, $R=2 / 1, r=1 / 2$ )

arithmetic "quarters"

$5 / 4,6 / 5,7 / 6,8 / 7$

III, iii, iiiL, T?

maj., min. and submin. thirds, supertone

thirds

C, E, G, Bk, C'

A.3.2. the perfect fifth $(\mathrm{V}, R=3 / 2, r=1 / 3)$

arithmetic "quarters"

$9 / 8,10 / 9,11 / 10,12 / 11$

$\mathrm{T}, \mathrm{t}, \mathrm{Tm}, \mathrm{Tw}$ (upwards) harmonic "quarters"

$8 / 7,7 / 6,6 / 5,5 / 4$

T7, iiiL, iii, III

supertone, submin., min. and maj.

C, D7, F, Ab, C'

(upwards) harmonic "quarters"

$12 / 11,11 / 10,10 / 9,9 / 8$

Tw, Tm, t, T 
4 tones: epogd., grave, epidec., ephendec. epogd.

$\mathrm{C}, \mathrm{D}, \mathrm{E}, \mathrm{F} \# \mathrm{~m}, \mathrm{G}$

A.3.3. the perfect fourth (IV, $R=4 / 3, r=1 / 4$ ) arithmetic "quarters"

$13 / 12,14 / 13,15 / 14,16 / 15$

Tn, TLu, $\Delta 7, \mathrm{~S}$

2 Avic. spondeia, superdiesis, just semitone spondeia

C, Dn, Ek, E, F

A.3.4. the (epogdoos) tone ( $\mathrm{T}, R=9 / 8, r=1 / 9$ )

arithmetic "quarters"

33/32, 34/33, 35/34, 36/35

$\mathrm{Sm}, \mathrm{Sw} \xi, \Delta \mathrm{L} \psi, \mathrm{I} 7$

4 quartertones: 3 Quintilianian, 1 Archytean

Quintilianian

$\mathrm{C}, \mathrm{D} b \mathrm{~m}, \mathrm{D} b \xi, \mathrm{DL}, \mathrm{D}$
4 tones: ephendec., epidec., grave,

C, Dw, Eb, F, G

(upwards) harmonic "quarters"

16/15, 15/14, 14/13, 13/12

$\mathrm{S}, \Delta 7, \mathrm{TL} \mathrm{u}, \mathrm{Tn}$

just semitone, superdiesis, 2 Avic.

C, Db, D 7, Eu, F

(upwards) harmonic "quarters"

36/35, 35/34, 34/33, 33/32

I $7, \Delta \mathrm{L} \psi, \mathrm{Sw} \xi, \mathrm{Sm}$

4 quartertones: 1 Archytean, 3

C, C7, C $\sharp \gamma, \mathrm{Dw}, \mathrm{D}$

\section{A.4. Quintisections}

\section{$k=5$ : arithmetic and (upwards) harmonic "fifth parts"}

A.4.1. the octave (VIII, $R=2 / 1, r=1 / 2$ )

arithmetic "fifth parts"

(upwards) harmonic "fifth parts"

6/5, 7/6, 8/7, 9/8, 10/9

10/9, 9/8, 8/7, 7/6, 6/5

iii, iiiL, T7, T, t

t, T, T7, iiiL, iii

an assortment of thirds and tones

an assortment of tones and thirds

$\mathrm{C}, \mathrm{E} b, \mathrm{G} b, \mathrm{~A} b, \mathrm{~B} b, \mathrm{C}^{\prime}$

A.4.2. the perfect fifth $(\mathrm{V}, R=3 / 2, r=1 / 3)$

arithmetic "fifth parts"

11/10, 12/11, 13/12, 14/13, 15/14

Tm, Tw, Tn, TLu, $\Delta 7$

an assortment of spondeia, superdiesis

spondeia

C, Dm, Eb, Fn, Ge, G

A.4.3. the perfect fourth (IV, $R=4 / 3, r=1 / 4$ )

arithmetic "fifth parts"

16/15, 17/16, 18/17, 19/18, 20/19

$\mathrm{S}, \mathrm{S} \xi, \Delta \psi, \mathrm{S} \gamma, \Delta \varphi$

5 miscellaneous half-tones

C, D-, E, F $\sharp, A, C^{\prime}$

$\mathrm{C}, \mathrm{D} b, \mathrm{E} b b \xi, \mathrm{E} b, \mathrm{~F} b \gamma, \mathrm{F}$

(upwards) harmonic "fifth parts" 15/14, 14/13, 13/12, 12/11, 11/10

$\Delta 7, \mathrm{TLu}, \mathrm{Tn}, \mathrm{Tw}, \mathrm{Tm}$

superdiesis, an assortment of

$C, C \exists, D \# u, E, F \# w, G$

(upwards) harmonic "fifth parts" 20/19, 19/18, 18/17, 17/16, 16/15

$\Delta \varphi, \mathrm{S} \gamma, \Delta \psi, \mathrm{S} \xi, \mathrm{S}$

5 miscellaneous half-tones ${ }^{35}$

$C, C \sharp \varphi, D-, D \sharp \psi, E, F$

\section{Appendix B: Notes on terminology and notation employed}

Several of the intervallic notations and terms used in this paper have been worked out by the author for purposes of theoretical convenience and are not or may not be generally recognized.

${ }^{35} \mathrm{Cf}$. footnote 14 . 
- "+" (plus or acute) and "-" (minus or grave) have their habitual meaning of a Didymean comma (81/80, roughly $1 / 9$ of a tone) sharper and flatter than the corresponding pitches in a just scale, respectively (e.g. F+, D-); the usual natural pitches are meant in the context of Zarlino's tuning; thus, "piano" A, E, D b, Eb and Bb are annotated, respectively, A+, E+, Db-, Eb- and $\mathrm{B} b-$.

- “ๆ” (super) and "L” (sub) are used for corresponding septimal pitches; in most cases they raise and lower the note, respectively, by roughly a quartertone; " "\#” (about three quartertones sharp) and " $\mathrm{b}$ " (about three quartertones flat) are meant to be called "supersharp" and "subflat", respectively.

- " $m$ " and "w" are used as alteration signs for undecimal notes; in most cases they both flatten the note by about a quartertone; "\#m" and "\#w" are compound undecimal alterations, both typically raising the note by about a quartertone; "bm" and "bw" are two other compound undecimal alterations, both typically lowering the note by about three quartertones;

- "n" and "u" are used as alteration signs for tridecimal notes in a way roughly similar to "m" and "w", causing slightly different flattening effects; the case with novemvigintesimal " $\varepsilon$ " and " $\mathrm{k}$ " and with untrentesimal " $\mathrm{p}$ " and " $\mathrm{q}$ " is also similar.

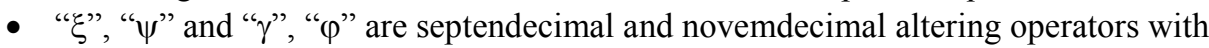
nearly negligible pitch-shifting effects.

- " $\theta$ " and " $\mathrm{l}$ " are trevigintesimal altering operators with slight (comma-sized) sharpening and flattening effects respectively.

\section{BIBLIOGRAPHY / WEBSITES}

[1] Aristoxenus, 1977, Harmonic elements vol. B, series: Ancient Harmonic Writers (Athens Greece, Georgiades publications - Library of the Hellenes) [in Greek].

[2] Boethius, Anicius Manlius Torquatus Severinus, De institutione musica liber III; manuscript: Cambridge, Trinity College, c. 505 a.D. [in Latin]. English Transl.: Bower Calvin D., 1989, Fundamentals of music (New Haven Connecticut, Yale University Press).

[3] Chrysanthus of Madytus, Archbishop of Dyrrhachium, 1832, Theoreticum magnum musicae (Trieste Italy, Michele Weis Typography) [in Greek].

[4] d'Erlanger, Baron Rodolphe, 1935/2001, La musique Arabe volumes 1-6, (Paris, Paul Geuthner) [in French].

[5] al-Fârâbî, Abû Nasr Mohammad ibn Farûkh, Kitâb al-mûsîqî al-kabir. Transl. into French as Grand traité de la musique. In: d'Erlanger vol. 1.

[6] Helmholtz, Hermann L.F., 1954, On the sensations of tone as a physiological basis for the theory of music, translated, revised, corrected and expanded by Ellis Alexander J. in 1885 ( $2^{\text {nd }}$ English edition New York: Dover Publications, Inc.) $\left[1^{\text {st }}\right.$ publication in German: 1863].

[7] ibn Sînâ (Avicenna), Abû 'Âli al-Husayn ibn 'Abd-Allâh, Kitâbu' š-šifâ'. Transl. into French as Avicenne mathématiques. In d'Erlanger vol. 2, 103-end.

[8] Jedrzejewski, Franck, Avicenne et le tempérament [in French]. Available online at: http://perso.club-internet.fr/daschour/micromegas/jedrzejewski/avicen.html (accessed 1 June 2006)

[9] Lekkas, Demetrios, 2003, "On the drilling of flutes", text \# 13 in the collective volume Accompanying texts for the course "Arts II: Overview of Greek music and dance" ELP 40, 1-9, (Patras Greece: The Hellenic Open University) [in Greek]. 
[10] Lekkas, Demetrios, 2006, "The diatonic basis of Byzantine music; systemic structural approach". In Polyphonia, 8, 7-35 (Athens Greece) [in Greek].

[11] Monzo, Joe, "Philolaus". Available online at: http://tonalsoft.com/enc/p/philolaus.aspx (accessed 1 June 2006)

[12] Nicomachus Gerasenus, Arithmētikè eisagōgē. Transl. into English in D’Ooge Martin Luther, 1926, Nicomachus of Gerasa, introduction to arithmetic, with notes by F.E.

Robbins and L.C. Karpinski, (New York: The MacMillan Co., repr. 1938, Ann Arbor: University of Michigan Press).

[13] Plato, Timceus, 1939, ancient text with introduction, translation (into Modern Greek) and comments by Th. Vlyziotis and Ch. E. Papanastasiou, Library of Ancient Greek Writers and Poets, (Athens Greece: Ioannis and P. Zacharopoulos) [in Greek; dialogue composed c. 360 b.C.].

[14] Ptolemy, Claudius, Harmonics. In Barker Andrew (Ed.), 1989, Greek musical writings vol. ii: "Harmonic and acoustic theory", ch. 11, 270-291, Cambridge Readings in the Literature of Music (Cambridge University Press) [in Greek].

[15] Rameau, Jean-Philippe, 1971, Treatise on harmony, translated, with an introduction and notes, by Philip Gossett (Dover Publications Inc.) [ $1^{\text {st }}$ publication in French: 1722].

[16] Sachs, Curt, 1943, The rise of music in the ancient world: east and west (New York: W.W. Norton).

[17] Schlesinger, Kathleen, 1939, The Greek aulos: a study of its mechanism and of its relation to the modal system of ancient Greek music, followed by a survey of the Greek harmoniai in survival or rebirth in folk-music (with an introduction by J.F. Mountford), (London: Methuen \& Co. Ltd.).

[18] Tannery, Paul (Ed.), 1940, Quadrivium de Georges Pachymère, texte revisé et établi par R.P. Elpidios Stéphanou A.A., Studi e Testi 94, (Città del Vaticano: Biblioteca Apostolica Vaticana) [in Greek].

[19] West, Martin L., 1992, Ancient Greek music (Oxford University Press).

Illustrated websites on the 7th millennium Neolithic flutes found in China, available online at:

http://www.shakuhachi.com/K-9KChineseFlutes-Nature.html (accessed 21 October 2018) http://www.bnl.gov/bnlweb/pubaf/pr/1999/bnlpr092299.html (accessed 21 October 2018)

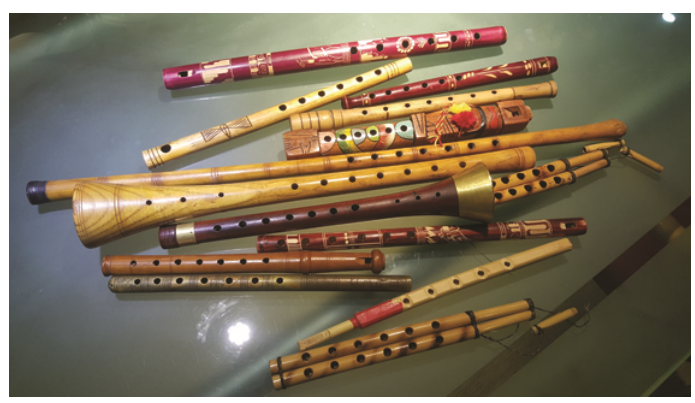

Assorted pipes with aliquot drillings from various continents and cultures. From the author's own collection. Photo: G. Pliagos 